\title{
Computer faceted thermal model of helicopter
}

\author{
by R. Dulski and M. Dąbrowski
}

Military University of Technology, Institute of Optoelectronics,

ul. Kaliskiego 2, 00-908 Warsaw, Poland, e-mail: rdulski@wat.waw.pl

\begin{abstract}
The paper presents a computer Faceted Thermal Target Model of helicopter (FTTM) describing radiative properties of target in the infrared. The model is predestined for target simulation in analyses of influence of thermal-scene conditions on the possibilities of target detection by a thermodetection system. This is a faceted model describing the target as a set of facets. The set of data for the model of helicopter requires information on the shape and dimensions of the target as well as on temperature distribution and emissivity coefficient of its surface. The model enables to determine emission of radiation from the target surface for various directions and distances of detection. It makes possible to obtain the data necessary to design devices for infrared recognition with a limited application of costly experimental investigations.
\end{abstract}

\section{Introduction}

In recent several years, intense research works on automatic target recognition (ATR) systems have been carried on in many research centres. Practical implementation of infrared radiation in the accomplishment of ATR goals is quite difficult since the radiation of targets is often similar to background radiation in the wavelength range detected (the background e.g. can include decoy targets). Thus, it is necessary to know thermal characteristic features called target signatures [1-4] enabling to distinguish the target from the background using detection systems. Expensive and complicated experimental investigations are often required to get the information indispensable to determine target signatures. Thermal characteristics of real (three-dimensional) targets depend essentially on the direction of detection. The determination of statistical measures of target thermal characteristics (essential to construct target recognition systems) requires large data files for various conditions of target detection. The target signatures depend on spatial distributions of temperature and emissivity coefficient of the surface. They depend also on the shape of the target and its orientation in relation to other sources of radiation.

Some of the above-mentioned parameters cannot be fully controlled, other often have a random character (e.g., the background radiation). The acquisition of the experimental data library for representative sets of the input parameters is a task requiring a great research and financial effort. Alternatives for experimental investigations are relatively inexpensive computer simulations, particularly modelling of a thermal scene.

Using the computer modelling, one can replace very costly experimental derivation of data sets necessary to determine thermal target signatures with low-cost and fast computations, which, moreover, enable controlled variation of the measuring conditions being simulated and constructional parameters of the device. A portion of the input data for computations can be obtained by means of simple experiments.

The results of computations can be used for design of a thermodetection system and can be helpful in interpreting of the sensor output signal.

\section{The model structure and assumptions}

The following assumptions have been made for construction of a Faceted Thermal Target Model (FTTM): 
- properties of the model are given on the target surface (the heat balance and heat circulation inside the target are not modelled),

- emissive properties of the surface are of Lambert lan character (i.e. a constant value of luminance is assumed in all directions),

- individual elements of the model have uniform temperature ( $T$ = const. for each facet),

- the emissivity coefficient of the target surface is constant ( = const). This simplification is admissible on the assumption of constant working conditions of the target and homogeneous covering of the whole surface),

- the target is a rigid solid (the facets constituting constructional subsystems of the target are immovable in relation to each other),

- the solar radiation as well as reflected radiation of the background and other objects is not considered.

Above simplifying assumptions in the theoretical model enables to reduce the amount of data necessary for computations and, thereby, speeds up computer simulations and lowers hardware requirements. In order to ensure maximum correspondence of the shape and dimensions of the model with those of the real target, a geometrical mesh of the model has been worked out on the basis of the technical documentation of the target being modelled. The temperature distribution on the surface of the model has been determined from the experimental thermal images of the real target.

Fig. 1 presents a schematic diagram of the data processing performed by the FTTM model. The model allows for generation of directional temperature and radiative characteristics of: the thermal scene including the target being modelled (the target and the background), the target alone, and the background alone.

Two-dimensional distributions of the radiance $(L(T))$ for a given location and orientation of the target and thermodetection system are obtained by transformation of two-dimensional distributions of temperature. The need for such a transformation results from the fact that optical imaging is linear in relation to luminance, but is not linear in relation to temperature.

The radiance $(L(T))$ is obtained by integrating the spectral density of luminance $\left(L_{\lambda}(T)\right)$ over the whole spectral region $(0, \infty)$. Spectral characteristics of the detection channel (the spectral coefficient of atmosphere transmission $\tau_{\mathrm{a}}(\lambda)$, the spectral coefficient of optics transmission $\tau_{0}(\lambda)$ and the spectral responsivity of detector $R_{\mathrm{n}}(\lambda)$ influence essentially the apparent thermal target signatures since they filter the spectrum of radiation emitted by the target. Thus, the apparent thermal target signatures do not result directly from the twodimensional distribution of radiance $(L(T(x, y)))$ but from the two-dimensional distribution of radiance detected $\left(L_{d}(T(x, y))\right)[5]$ which is defined as:

$$
L_{d}(T)=C_{1} \int_{\lambda_{1}}^{\lambda_{2}} L_{\lambda}(T) \tau_{a}(\lambda) \tau_{o}(\lambda) R_{n}(\lambda) d \lambda
$$

The spectral coefficient of atmosphere transmission $\left(\tau_{\mathrm{a}}(\lambda)\right)$ was computed using the LOWTRAN database.

From the product of above functions, one can conclude on relative contribution of radiation of a selected wavelength in generation of the output signal of the detector.

\section{Input data for the model}

The computer geometrical model of a three-dimensional target has been built from quadrilateral facets. A technical documentation for the target being modelled can be read into CAD-type (Computer Aided Design) programs. The data read in, however, require conversion (reducing the number of facets, replacing convex or concave surfaces with flat ones, approximation of facet shapes with quadrilaterals). Fig. 2 presents an example of the geometrical mesh of target (helicopter) created with the use of the AutoCAD program. 


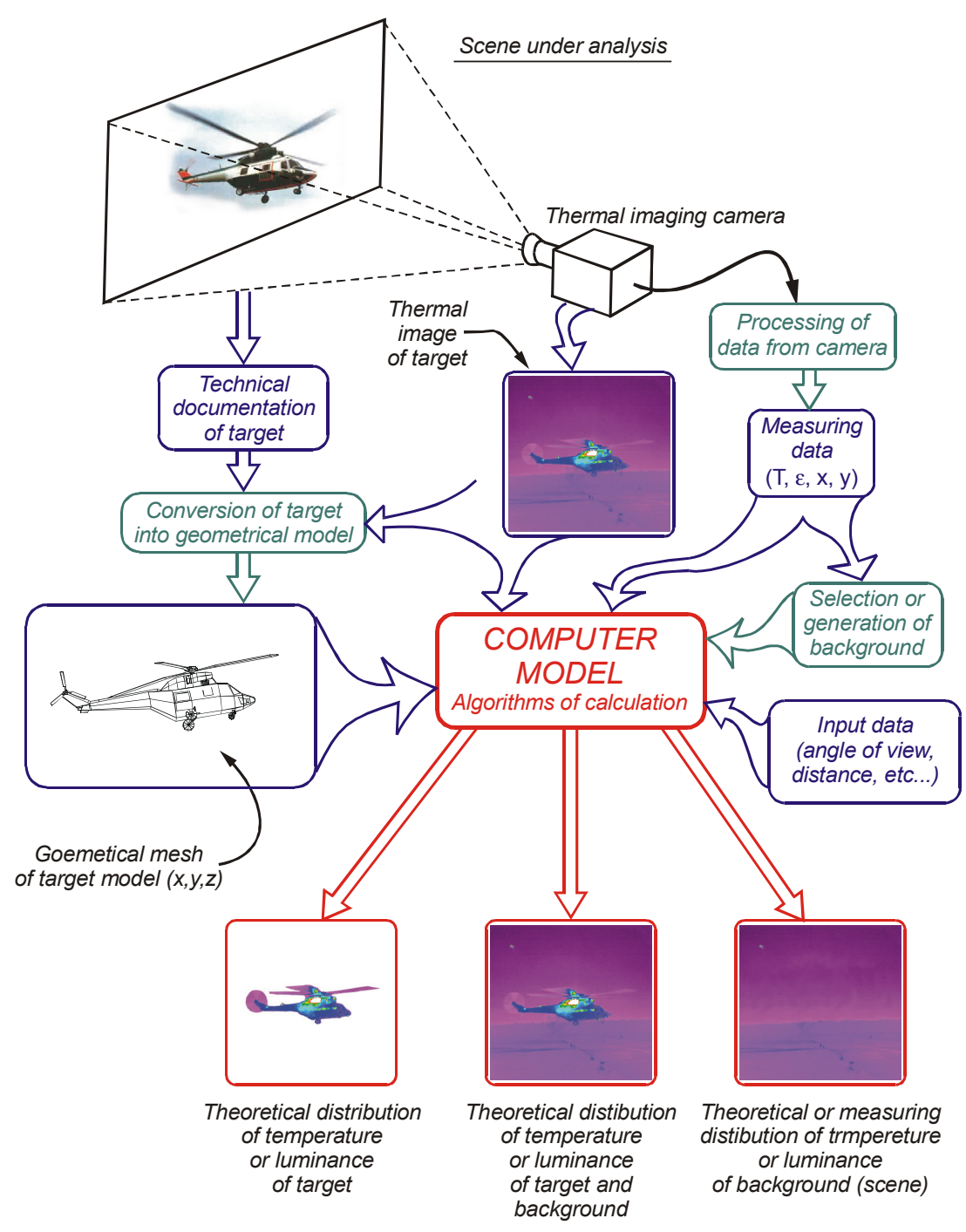

Fig. 1. Schematic diagram of data processing performed by FTTM simulation program

The way of segmentation of the target modelled into facets and their number are significant for the correspondence of thermal properties of the target model and the real target. For instance, the regions of the target surface having crucial influence on its thermal properties (e.g., those of high heat contrast or of temperature considerably different from the average target temperature) should be divided into a higher number of facets.

Using the AutoCAD program, a list of the model elements has been created and transformed to the form required by the FTTM simulation program accomplishing the model. 


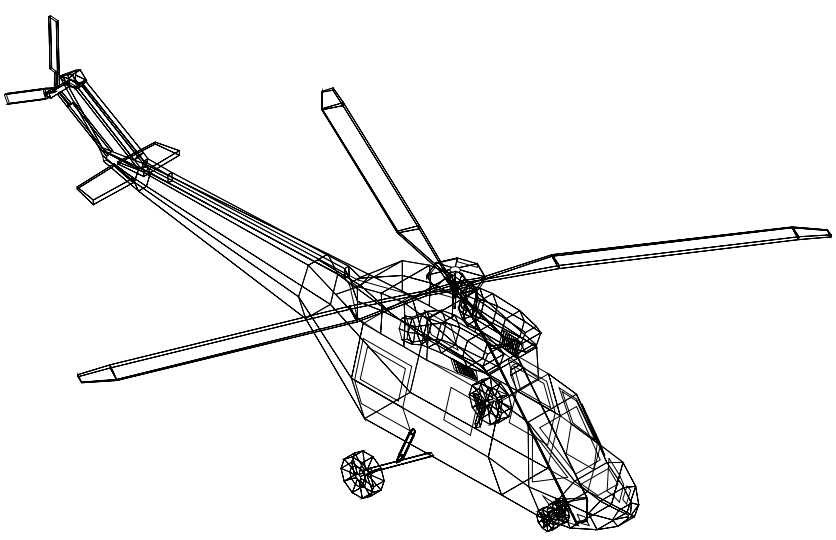

Fig. 2. Geometrical mesh of a sample helicopter

The last element in the data file of the target are temperature and coefficients of emissivity assigned to the facets. Temperature of the model facets was determined from the thermograms taken with the measuring thermal imaging system. For this purpose, thermograms registered during standard experimental investigations from a short distance were used. Exemplary thermogram used to assign temperatures to the facets of the geometrical model of the target is presented in fig. 3 (on the right). The thermograms recorded digitally were converted to temperature matrices. Using the Spyglass Transform program the averaged values of temperatures were rewritten, in the regions pointed out of the target surface and were assigned to the facets of the target model corresponding to those regions. From experimental investigations, a constant averaged coefficient of emissivity of the target facets was assumed.

The targets were located on a thermal background during the modelling process. The data about the background were obtained from the thermograms registered with a thermovision camera from a long distance (fig. 3 on the left), by their transformation to temperature matrices [6].
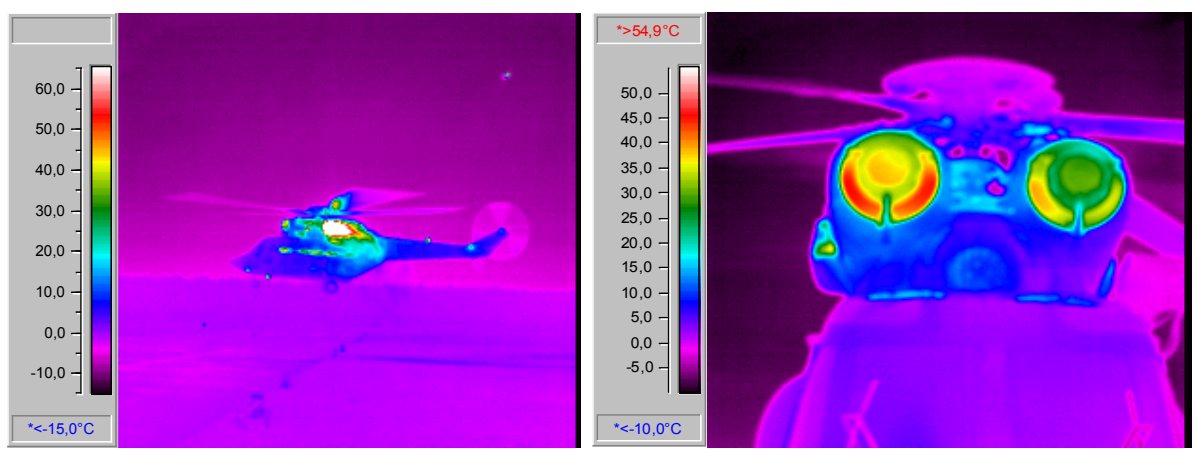

Fig. 3. Sample thermograms registered during experimental investigations 


\section{Accomplishment of the thermal target model}

The simulation program of the thermal target (FTTM) developed originally by the authors of this paper allows to determine two-dimensional geometrical images of the target and background for an arbitrary angle of the field of view, direction of detection, and location of the thermodetection system in relation to the target. The information about temperature and emissivity coefficient of the target facets and background is projected onto these images. The two-dimensional distributions of temperature and coefficient of emissivity are sampled and recorded in matrices in a given manner.

The input data for the FTTM program are the target and background. Two-dimensional geometrical images of the target and background are determined in the rectangle of analysis of the camera image. Here, the name "camera" stands for the thermodetection system to emphasize that the program supplies data for the image analysis. The field of view of the camera and the rectangle of image analysis are selected much larger than the field of view of the thermodetection system under investigation.

The rectangle of image analysis is divided into $256 \times 256$ equidistant points. A ray originating from the centre of the camera is traced through each point. The data being determined are: the point of crossing the target facet or terrain, the angle at which the crossing occurs, and the temperature $(T)$ of the target at the crossing point. If the ray crosses only the terrain, the temperature is determined using the interpolation of temperatures of adjacent points corresponding to respective elements of the matrix with the data about the terrain. If the ray crosses a few facets of the target, the nearest facet is selected, and the temperature and coefficient of emissivity are determined from the temperature and emission coefficient of this facet.

The results of computations (two-dimensional distributions of temperature or radiance) are typically recorded in 256×256-element matrices, but matrices of larger dimension can also be used. The standard assumed here results from capacity of the RAM and the operating speed of computer applied for simulations.

Fig. 4 presents examples of thermograms generated by means of the FTTM program. The colour scale is denoted in kelvins.

\section{Conclusions}

A faceted model of a three-dimensional thermal target has been proposed which describes well radiative properties of helicopter in the infrared spectral region. The model requires the data on shape and dimensions of the target as well as on distribution of temperature and emissivity of the surface.

The presented computer model well approximates the real three-dimensional thermal target when external thermal sources are omitted.

It has been proved that the computer modelling can essentially reduce the experimental costs of evaluation of the thermal target signatures.

\section{Acknowledgments}

This research was performed under the support of the State Committee for Scientific Research of the Republic of Poland (program no. T00A 008 23).

\section{REFERENCES}

[1] KLEIN, L.A., "Millimeter-Wave and Infrared Multisensor Design and Signal Processing", Artech House, Boston 1997

[2] LIOYD, J.M., "Thermal Imaging Systems", Plenum Press, New York 1975

[3] SEYRAFI, K. and HOVANESSIAN, S.A., "Introduction to Electro-Optical Imaging and Tracking Systems", Artech House, Boston 1993 
[4] ASCETA, J.S., SHUMAKER, D.L., eds., "The Infrared and Electro-Optical Systems Handbook", Vol.4 Electro-Optical Systems Design, Analysis, and Testing, SPIE Optical Engineering Press, Bellingham 1993

[5] DULSKI, R., "A method for determining of thermal imaging of three-dimensional targets and its experimental verification", Doctoral Thesis, The Main Library of the Military University of Technology, Warsaw, 1998, (in Polish)

[6] WATKINS, W.R., CLEMENT, D., eds., "Characterization, propagation, and simulation of sources and backgrounds", Proceedings of the SPIE 1486, 1991

a)
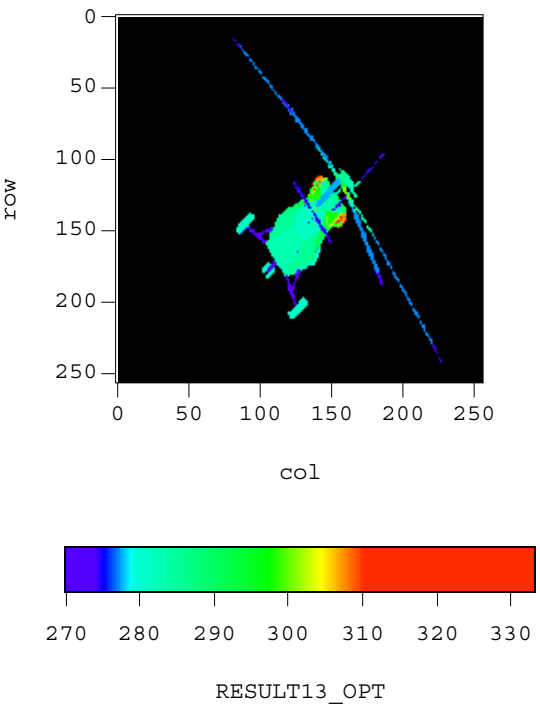

b)

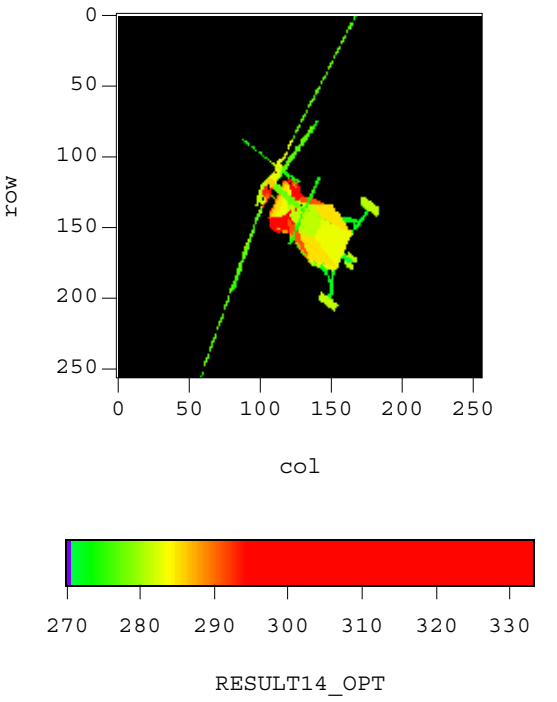

Fig. 4. Computer-generated thermal images (using FTTM program) for two different detection situations 